\title{
CORRELATION OF MEAN PLATELET VOLUME AND PLATELET DISTRIBUTION WIDTH IN RISK CATEGORIES OF DENGUE FEVER- A PILOT STUDY
}

\author{
Nidhish Kumar1, Mayuri Swamy², Shrijeet Chakraborti ${ }^{3}$, Jyothi Kini ${ }^{4}$, Tanwi Singh 5 , Bhawana Sahay 6 , Sharvani Singh ${ }^{7}$ \\ 1 Senior Resident, Department of Transfusion Medicine and Blood Bank, All India Institute of Medical Sciences, Patna. \\ ${ }^{2}$ Tutor, Department of Pathology, Kasturba Medical College, Mangalore. \\ ${ }^{3}$ Associate Professor, Department of Pathology, Kasturba Medical College, Mangalore. \\ ${ }^{4}$ Associate Professor, Department of Pathology, Kasturba Medical College, Mangalore. \\ ${ }_{5}^{5}$ Senior Resident, Department of Transfusion Medicine and Blood Bank, All India Institute of Medical Sciences, Patna. \\ ${ }^{6}$ Senior Resident, Department of Transfusion Medicine and Blood Bank, All India Institute of Medical Sciences, Patna. \\ 7Tutor, Department of Pathology, RMC Pravara Institute of Medical Sciences, Loni.
}

\section{BACKGROUND}

ABSTRACT

Incidence of dengue fever is on rise in India. We investigated the characteristics of Mean Platelet Volume (MPV) and platelet distribution width (PDW) in various risk categories of dengue fever.

\section{MATERIALS AND METHODS}

In this prospective two months pilot study, 100 cases of NS1 antigen and IgM ELISA positive dengue were included. Platelet count for risk categorisation, MPV and PDW values were recorded. Statistical analysis was done using ANOVA (Fisher's test).

\section{RESULTS}

There were $77 \%, 18 \%$ and 5\% cases in no, low and moderate risk categories, respectively. In no, low and moderate risk categories, mean MPV was 8.6, 9.6 and 10.7 (fL), respectively. In no, low and moderate risk categories mean PDW was 16.4, 17.03 and 16.9, respectively. Overall, there was positive correlation between MPV and PDW ( $p=0.066 ; r=0.185)$, as also in low risk category. In moderate risk group, there was a negative correlation between MPV and PDW $(p=0.201 ; r=-0.686)$.

\section{CONCLUSION}

Hence, a positive correlation was seen between MPV and PDW in low risk group, but no correlation was seen in no risk group.

\section{KEYWORDS}

Dengue Fever (DF), Dengue Haemorrhagic Fever (DHF), Mean Platelet Volume (MPV), Platelet Distribution Width (PDW), Plateletcrit (PCT), Non-Structural Protein 1 (NS1).

HOW TO CITE THIS ARTICLE: Kumar N, Swamy M, Chakraborti S, et al. Correlation of mean platelet volume and platelet distribution width in risk categories of dengue fever- a pilot study. J. Evolution Med. Dent. Sci. 2018;7(02):142-145, DOI: $10.14260 /$ jemds/2018/31

\section{BACKGROUND}

Dengue is the most prevalent mosquito-borne viral infection worldwide with 100 million Dengue Fever (DF) cases and half a million cases of Dengue Haemorrhagic Fever (DHF) annually.(1,2) Incidence of dengue fever is on rise in India. There have been regular epidemics of DHF in India with increase in number of adult dengue patients with both significant morbidity and increasing number of adult deaths due to dengue.(1,2)

Dengue fever is an acute febrile illness and has a wide spectrum of clinical presentations with unpredictable clinical outcome. Majority of patients have self-limiting clinical course, only a small proportion of patients progress to DHF when they acquire second infection by a different dengue viral antigen.(3)

'Financial or Other Competing Interest': None.

Submission 29-11-2017, Peer Review 23-12-2017,

Acceptance 29-12-2017, Published 08-01-2018.

Corresponding Author:

Dr. Nidhish Kumar,

C/o. Dr. Mohan Singh,

Jagat Narayan Road,

Kadam Kuan, Opposite Indra Lok Apartment,

Patna-80003, Bihar.

E-mail:drnidhishkumar@gmail.com

DOI: $10.14260 /$ jemds $/ 2018 / 31$
The World Health Organisation (WHO) classifies the clinical course of DHF into four grades based on laboratory studies: Grade I- Fever with positive tourniquet test; Grade IIPlus mild spontaneous bleeding; Grade III- Presence of weak and rapid pulse; Grade IV- Profound shock with undetectable pulse. The last two are considered as Dengue Shock Syndrome (DSS).(4)

The primary dengue vector Aedes aegypti mosquito has spread widely in tropical and subtropical regions. Dengue fever virus (DENV) is an RNA virus of the family Flaviviridae and is divided into four serotypes, DENV 1 - 4.(5) Detection of dengue specific antigen is the mainstay of diagnosis of dengue infection. Antibody detection is an indirect method of diagnosis and often gives false positive as well as false negative results. Non-structural protein 1 (NS1) is used to detection of dengue viral antigen and is sensitive as well as highly specific.(6)

Severity of illness is determined by various risk factors such as age, pre-existing illness, infecting serotype and secondary infection.(7) The most common laboratory finding in DF is thrombocytopenia.(8) The mechanism of thrombocytopenia remains unclear in DF. Possible mechanisms of thrombocytopenia in DF may be due to direct bone marrow suppression by the virus; anti-dengue antibody mediated platelet destruction, peripheral consumption of platelets and isolated viral replication in the platelet. $\left.{ }^{9}\right)$ 
Recently, Platelet Indices- Mean Platelet Volume (MPV), Platelet Distribution Width (PDW) and Platelet large cell ratio (P-LCR) have been investigated as prospective platelet activation markers.(10) Platelet volume is calculated as MPV and is a surrogate marker for bone marrow activity; increased megakaryocytic activity is indicated by high MPV values. A low MPV value is seen in marrow suppression and is associated with increased risk of bleeding.(11) Platelet activation alters the morphology of these cells, which is evaluated on the basis of MPV and PDW.(10) Plateletcrit (PCT) another platelet parameter which is used as a reliable measurement for platelet biomass, as it combines both MPV with absolute platelet count.(12) Platelet large cell ratio (PLCR) is significantly decreased in thrombocytosis and increased in thrombocytopenia; P-LCR inversely related to platelet count and directly related to PDW and MPV.(13)

The aim of our study was to investigate the characteristics of mean platelet volume (MPV) and platelet distribution width (PDW) in various risk categories of dengue fever.

\section{MATERIALS AND METHODS}

This is an observational study and conducted prospectively for a period of two months as a pilot study from September 2014 to October 2014 at Department of Pathology, Kasturba Medical College, Mangalore. Total 100 cases were included in the study, which were divided into no risk (platelet count > 1,00,000/cu.mm), low risk (platelet count 1,00,000 $50,000 /$ cu.mm), moderate risk (platelet count 20,000 50,000 /cu.mm) and high risk (platelet count < $20,000 /$ cu.mm) categories based on platelet count. The inclusion criteria were all the patients with clinical symptoms of dengue and serologically dengue positive cases. The exclusion criteria include patients with serologically negative dengue and laboratory findings which suggested a bacterial, parasite or any viral infection excluding dengue infection or any other disease. The platelet parameters platelet count (PLT), MPV, PDW and PCT were measured by automated cell counter Beckman Coulter LH780. The dengue NS1 antigen was detected by antigen-antibody reaction principle, which is a rapid visual test for dengue.

Data was entered in Microsoft Office Excel Sheet 2010. Statistical analysis was done using SSPS version 20 software. The results were obtained using Fisher's exact test, intergroup comparisons were done using ANOVA and correlations between the groups were done using Pearson's correlation formula. A ' $p$ ' value of $<0.05$ was considered significant.

\section{RESULTS}

This is a prospective study. 100 cases of serologically positive dengue patients were studied, who had clinical features of dengue infection. The 100 cases were divided according to high, moderate, low and no risk categories based on platelet count. There were 77 cases in 'no risk' group, 18 cases in 'low risk' and 5 cases in 'moderate risk.' There were no cases in high risk category. Median MPV was 8.5 (range 6.2 - $14.9 \mathrm{fL}$ ), median PDW was 16.5 (range 15.2 - $18.6 \mathrm{fL}$ ), median PLT was $140 \times 10^{3}$ (range $18 \times 10^{3}-632 \times 10^{3} / \mu \mathrm{L}$ ) and median PCT was 0.125 (range 0.021 - 0.427). On statistical analysis, significant correlation was seen for PDW among the different risk categories $(F=7.954 ; p=0.001)$. No correlation was seen between MPV and PDW in no risk and low risk categories, whereas negative correlation was observed in moderate risk category. No correlation was seen for MPV among the different risk categories $(F=3.489 ; \mathrm{p}=0.034)$. The 'No risk' group showed negative correlation between MPV and platelet count, and PDW and platelet count. The 'Low risk' group showed negative correlation between PDW and platelet count. The 'Moderate risk' group showed negative correlation between MPV and platelet count.

The correlation values of MPV and PDW levels among high, low and no risk groups is depicted in Table 1 and 2 . Categorisation of 100 cases among moderate, low and no risk groups is depicted in the pie chart. The bar diagrams 1 and 2 depict the MPV levels with its respective mean value and PDW levels with its mean values.

\section{CATEGORIZATION OF CASES}

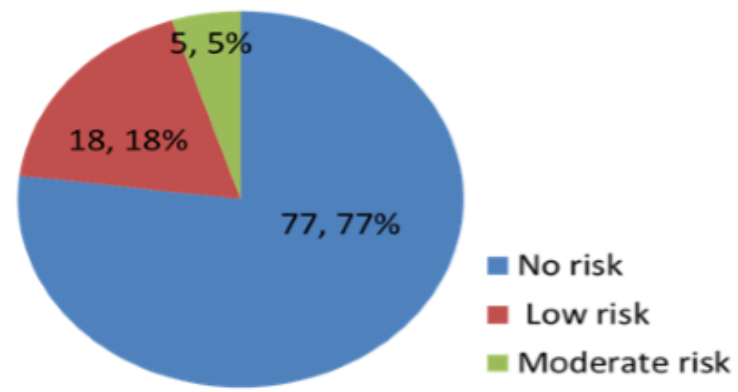

Fig.1. Total Number of Cases $(\mathrm{N})=100$
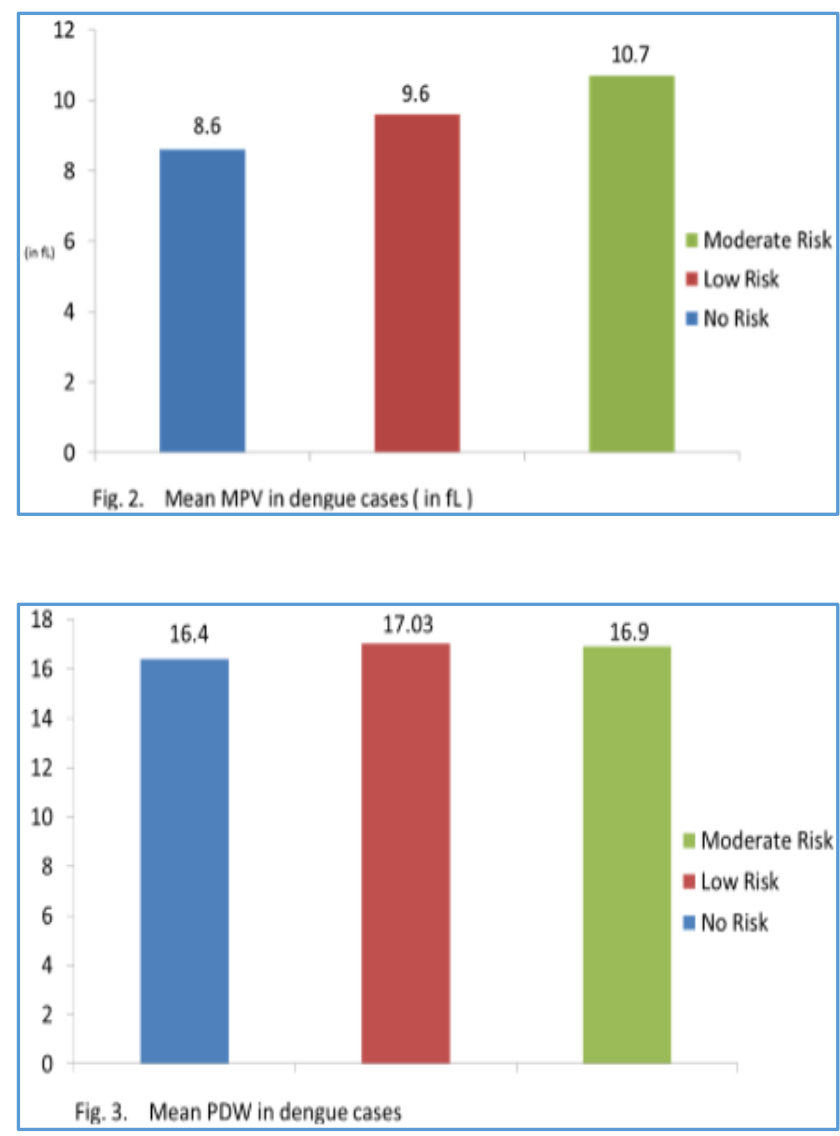


\begin{tabular}{|c|c|c|c|c|c|}
\hline \multicolumn{6}{|c|}{ Correlations } \\
\hline \multicolumn{3}{|c|}{ Groups } & MPV & PDW & PLT \\
\hline \multirow{9}{*}{ No Risk } & \multirow{3}{*}{$\mathrm{TC}$} & $r$ & -.220 & .103 & .575 \\
\hline & & p & .054 & .374 & .000 \\
\hline & & $\mathrm{N}$ & 77 & 77 & 77 \\
\hline & \multirow{3}{*}{ MPV } & $\mathrm{r}$ & & .086 & -.492 \\
\hline & & $p$ & & .456 & .000 \\
\hline & & $\mathrm{N}$ & & 77 & 77 \\
\hline & \multirow{3}{*}{ PDW } & $\mathrm{r}$ & & & -.077 \\
\hline & & $p$ & & & .505 \\
\hline & & $\mathrm{N}$ & & & 77 \\
\hline \multirow{9}{*}{ Low Risk } & \multirow{3}{*}{ TC } & $\mathrm{r}$ & .208 & .496 & -.226 \\
\hline & & $p$ & .408 & .036 & .367 \\
\hline & & $\mathrm{N}$ & 18 & 18 & 18 \\
\hline & \multirow{3}{*}{ MPV } & $\mathrm{r}$ & & .408 & .056 \\
\hline & & $\mathrm{p}$ & & .093 & .826 \\
\hline & & $\mathrm{N}$ & & 18 & 18 \\
\hline & \multirow{3}{*}{ PDW } & $\mathrm{r}$ & & & -.351 \\
\hline & & $\mathrm{p}$ & & & .153 \\
\hline & & $\mathrm{N}$ & & & 18 \\
\hline \multirow{9}{*}{$\begin{array}{c}\text { Moderate } \\
\text { Risk }\end{array}$} & \multirow{3}{*}{ TC } & $\mathrm{r}$ & .091 & -.732 & -.817 \\
\hline & & $\mathrm{p}$ & .885 & .160 & .092 \\
\hline & & $\mathrm{N}$ & 5 & 5 & 5 \\
\hline & \multirow{3}{*}{ MPV } & $\mathrm{r}$ & & -.686 & -.323 \\
\hline & & $\mathrm{p}$ & & .201 & .596 \\
\hline & & $\mathrm{N}$ & & 5 & 5 \\
\hline & \multirow{3}{*}{ PDW } & $\mathrm{r}$ & & & .699 \\
\hline & & $\mathrm{p}$ & & & .189 \\
\hline & & $\mathrm{N}$ & & & 5 \\
\hline \multicolumn{6}{|c|}{ Table 1} \\
\hline
\end{tabular}

\begin{tabular}{|c|c|c|c|}
\hline \multicolumn{4}{|c|}{ Correlations } \\
\hline Group & & & PDW \\
\hline \multirow{3}{*}{ No Risk } & \multirow{3}{*}{ MPV } & $\mathrm{r}$ & .086 \\
\hline & & $\mathrm{p}$ & .456 \\
\hline & & $\mathrm{N}$ & 77 \\
\hline \multirow{3}{*}{ Low Risk } & \multirow{3}{*}{ MPV } & $r$ & .408 \\
\hline & & $\mathrm{p}$ & .093 \\
\hline & & $\mathrm{N}$ & 18 \\
\hline \multirow{3}{*}{ High Risk } & \multirow{3}{*}{ MPV } & $\mathrm{r}$ & -.686 \\
\hline & & $\mathrm{p}$ & .201 \\
\hline & & $\mathrm{N}$ & 5 \\
\hline
\end{tabular}

\begin{tabular}{|c|c|}
\hline $\begin{array}{c}\text { Categories of } \\
\text { Dengue Cases }\end{array}$ & $\begin{array}{c}\text { Correlation: MPV and } \\
\text { PDW }\end{array}$ \\
\hline No Risk & None \\
\hline Low Risk & Positive* $^{*}$ \\
\hline Moderate Risk & Negative** $^{* *}$ \\
\hline${ }^{*} \mathrm{p}=0.066 ; \mathrm{r}=0.185$ & \\
$* * \mathrm{p}=0.201 ; \mathrm{r}=-0.686$ & Table 3 \\
\hline \multicolumn{2}{|c|}{} \\
\hline
\end{tabular}

\section{DISCUSSION}

Dengue fever is a self-limiting febrile illness, whereas DHF is characterised by prominent haemorrhagic manifestations associated with thrombocytopenia and an increased vascular permeability. The onset of thrombocytopenia can be due to many factors. It can result due to immune response against platelet to decreased platelet production. It can also result due to direct infection of megakaryocytes by dengue virus, which leads to increased destruction of platelets. In acute stage of dengue fever, thrombocytopenia is observed due to bone marrow depression.(14) There are only few studies investigating the significance of changes in platelet indices during dengue infection. Mean platelet volume has been evaluated as a diagnostic tool to differentiate various conditions associated with thrombocytopenia such as to discriminate aplastic anaemia, hypoproductive thrombocytopenia, bone marrow disease and bone marrow metastatic tumours, but it has limited specificity and sensitivity.(11,13,15) PDW is a measure of platelet anisocytosis, and the "plateletcrit" which is the product of the MPV and platelet count. The PDW has been found to be of some use in distinguishing essential thrombocythemia (increased PDW) from reactive thrombocytosis (PDW normal). The PCT does not appear to provide any information of clinical value.(13)

In our study, we found that MPV values increased with decreased platelet count, but PDW had no correlation with decreasing platelet count. Our analysis showed significant correlation of PDW seen among different risk categories. There was no significant correlation between MPV and PDW.

The changes in MPV level in dengue fever was studied by Wiwanikit V and was observed that MPV in patients with DHF was not decreased and was similar as in healthy population.(11) A study by Bashir $\mathrm{AB}$ et al observed that patients with dengue fever had lower levels of MPV and platelet count; however, PDW values were increased in patients with dengue fever.(14) Another approach was adopted by Kurata et al to assess reticulated platelet using RNA-binding dyes and flow cytometric analysers, as these reticulated platelets are newly released from the bone marrow which can help in determining the aetiology of thrombocytopenia.(16)

The platelet parameters were also studied by Navya BN et al. They observed that platelet count as predictive parameter of DF/DHF/DSS and they also showed that low MPV and high PDW sensitivity for dengue fever.(17) A study conducted by Hardeva RN et al recorded significant association between platelet counts and severity of the disease. Low platelet count, low MPV, low PCT, high PDW and high P-LCR shows considerable sensitivity and specificity for dengue fever and can be used as a predictor of severity of dengue infection. ${ }^{(18)}$

\section{CONCLUSION}

In conclusion, significant differences were observed in the MPV, PDW, PCT and PLT. They showed that MPV level increased as the platelet count decreased, but PDW has no correlation with the decrease in platelet count. Hence, MPV and PDW have no value in predicting Dengue infection.

\section{REFERENCES}

[1] Swash M. Hutchinson's clinical methods. 20 th edn. Philadelphia: Bailliere Tindall 1995.

[2] Pruthvi D, Shashikala P, Shenoy V. Evaluation of platelet count in dengue fever along with seasonal variation of dengue infection. J Blood Disorders Transf 2012;3:128.

[3] Ramos MM, Tomashek KM, Arguello DF, et al. Early clinical features of dengue infection in Puerto Rico. Trans R Soc Trop Med Hyg 2009;103(9):878-84.

[4] WHO, Dengue: Guidelines for Diagnosis, Treatment, Prevention and Control, WHO, Geneva, \Switzerland, 2009.

http://whqlibdoc.who.int/publications/2009/978924 1547871 eng.pdf. 
[5] Mackenzie JS, Gubler DJ, Petersen LR. Emerging flaviviruses: the spread and resurgence of Japanese encephalitis, West Nile and dengue viruses. Nat Med 2004;10(12 Suppl):S98-109.

[6] Aruna R, Prakash M. A comparative study on dengue virus infection: serological markers Vs platelet count. Int J Curr Rev Aca Rev 2014;2(12):232-5.

[7] Guzman MG, Halstead SB, Artsob H, et al. Dengue: a continuing global thread. Nat Rev Microbiol 2010;8(12 Suppl):S7-16.

[8] Chuang YC, Lin YS, Liu CC, et al. Factors contributing to the disturbance of coagulation and fibrinolysis in dengue virus infection. J Formos Med Assoc 2013;112(1):12-7.

[9] Gupta E, Dar L, Kapoor G, et al. The changing epidemiology of dengue in Delhi, India. Virol J 2006;3:92.

[10] Vagdatli E, Gounari E, Lazaridou E, et al. Platelet distribution width: a simple, practical and specific marker of activation of coagulation. Hippokratia 2010;14(1):28-32.

[11] Wiwanikit V. Mean platelet volume in the patients with dengue hemorrhagic fever. Platelets 2004;15(3):185.

[12] Greisenegger S, Endler G, Hsieh K, et al. Is elevated mean platelet volume associated with a worse outcome in patients with acute ischemic cerebrovascular events? Stroke 2004;35(7):1688-91.
[13] Babu E, Basu D. Platelet large cell ratio in the differential diagnosis of abnormal platelet counts. Indian J Pathol Microbiol 2004;47(2):202-5.

[14] Bashir AB, Saeed OK, Mohammed BA, et al. Thrombocytopenia and bleeding manifestation among patients with dengue virus infection in Port Sudan, red sea state of Sudan. J Infect Dis Immun 2015;7(2):7-13.

[15] Kaito K, Otsubo H, Usui N, et al. Platelet size deviation width, platelet large cell ratio, and mean platelet volume have sufficient sensitivity and specificity in the diagnosis of immune thrombocytopenia. Br J Haematol 2005;128(5):698-702.

[16] Kurata Y, Hayashi S, Kiyoi T, et al. Diagnostic value of tests for reticulated platelets, plasma glycocalcin and thrombopoietin levels for discriminating between hyperdestructive and hypoplastic thrombocytopenia. Am J Clin Pathol 2001;115(5):656-64.

[17] Navya BN, Patil S, Kariappa TM. Role of platelet parameters in dengue positive cases-an observational study. Int J Health Sci Res 2016;6(6):74-8.

[18] Hardeva RN, Shyam LM, Sahil P, et al. Evaluation of platelet indices in patients with dengue infection. Int J Sci Res 2016;5(7):78-81. 